\title{
Experiencias fuera del cuerpo: una evaluación del constructo de transliminalidad y límite "fino" como anomalía cognitivo-perceptual
}

Out of body experiences: an evaluation of the construct of transliminality and "thin" boundaries as cognitive-perceptual anomaly

\section{Experiencias fora del cuerpo: una evaluação do construto de transliminalidad e limite "fino" como anomália cognitivo-perceptual}

\author{
Alejandro Enrique Parra \\ Universidad Abierta Interamericana, Argentina
}

Autor referente: rapp@fibertel.com.ar

Historia Editorial:

Recibido: 03/12/2016

Aceptado: 16/02/2018

\section{RESUMEN}

La experiencia fuera del cuerpo (EFC), se define como una experiencia en la cual el "yo", o el centro de conciencia, parece ocupar una posición remota respecto a su propio cuerpo. Un problema en la evaluación de ciertas anomalías perceptuales que se extrapolan del contexto de la psiquiatría clínica es su excesiva dependencia a ciertos fenómenos alucinatorios. La hipótesis de la transliminalidad sugiere que la fuente inmediata de nuestras percepciones se procesa primero a un nivel inconsciente, luego a través del umbral de la conciencia. El constructo de "límite" permite comprender ciertos factores que subyacen en la variedad de las experiencias excepcionales,

como las experiencias fuera del cuerpo. Se ponen a prueba tres hipótesis específicas: (1) personas que tienen experiencias fuera del cuerpo (EFC) tienen una mayor frecuencia de experiencias cognitivas anómalas (2) son más transliminales, (3) y tienen límites más "finos" en comparación con un grupo control (sin EFC). Cien individuos con EFC (47 \%) fueron comparados con otros 111 que no tuvieron EFC (53 \%), con un rango etario entre 18 a 83 años $(M=44,92$; $D T=13,29)$. Individuos con EFC puntuaron más alto en experiencias anómalas, límites más finos, y alta transliminalidad, lo cual confirman las hipótesis. Este estudio concluye que 
las experiencias fuera del cuerpo representan la sensibilidad de un individuo debido a los límites permeables del yo. Esta sensibilidad, relacionada con ciertas diferencias fisiológicas en el procesamiento perceptual, puede también representar una forma de transliminalidad.

Palabras clave: Experiencia fuera del cuerpo; Transliminalidad; Límites finos; Experiencias anómalas

\section{ABSTRACT}

Out-of-body experience (OBE) is an experience in which the "self", or center of awareness, seems to the person having the OBE to temporarily occupy a position spatially remote from the body. A drawback of assessing perceptual anomalies by extrapolating exclusively from the context of clinical psychiatry is the overreliance on hallucinatory phenomena. Transliminality hypothesis suggests that the immediate source of our perceptions is not our eyes or our ears, but rather the subliminal consciousness: percepts are first processed at an unconscious level and then, usually speedily, they are presented across the threshold to consciousness. The boundary construct is highly valuable in terms of understanding the factors which underpin the varieties of exceptional experiences, such as out of body experiences. Three specific hypotheses are tested here: (1) people who report OBEs (experients) have a higher capacity for cognitive anomalous experiences (2) higher transliminality, (3) and thinner boundaries that score differently than control (non-experients). Participants who experienced OBEs $(n=100,47 \%)$ were matched with participants who do not report OBEs (non experient, $\mathrm{n}=111,53 \%$ ), ages ranged from 18 to 83 years old ( $M=$ 44.92; $S D=13.29$ ). OBErs scored higher on anomalous experiences, higher on "thin" boundaries, high transliminality than for non-OBErs, which supported the three hypotheses. The paper discusses OBE phenomena as an experient's sensitivity due to permeable ego boundaries. This sensitivity, may be related to some physiological differences in perceptual processing may also underly it.

Keywords: Out-of-body experience; Transliminality; Thin boundaries; Anomalous experiences

\section{RESUMO}

A experiência fora do corpo (EFC), se define como uma experiência na qual o "yo", o centro de consciência, parece ocupar uma posição remota quanto ao seu próprio corpo. Um problema na avaliação de certas anomalias perceptuelles que se extrapolam do contexto da psiquiatría clínica é su excesiva dependencia a ciertos fenómenos alucinatorios. A hipótese da tradução é sugerida por uma fonte imediata de nossas percepções se processar primeiro a um nível inconsciente, em seguida, através do umbral da consciência. $O$ construto de "limite" permite compreender certos fatores que subentran na variedade de experiências excepcionais, como as experiências fora do corpo. (1) Penseis que tenham experiências fora do corpo (EFC) têm uma previsão de experiências cognitivas anómalas (2) son más transliminales, (3) e têm limites mais "finos" em comparação com un controle de grupo (sin EFC). Cien nos com EFC (47\%) foram comparados com outros 111 que no tuvent EFC (53\%), com um rango etario 
entre 18 a 83 años ( $M=44,92$; DT = 13,29). Individuos con EFC puntuaron más alto em experiências anómalas, limites mais finos, e alta transliminalidad, lo cual confirman as hipótesis. Este estudo conclui que as experiências fora do corpo representam a sensibilidade de um indivíduo a um limite de limites permeáveis do yo. Esta sensibilidade, relacionada com outras diferenças fisiológicas no processo de percepção, também é representativa de uma tradução de tradução.

Palavras-chave: Experiencia fuera del cuerpo; Transliminalidad; Límites finos; Experiencias anómalas

\section{Introducción}

rwin (2004) definió una "experiencia fuera del cuerpo" (EFC) como un experiencia mediante la cual el "yo", o centro de la conciencia, parece ocupar -para quien la experimenta- una posición remota respecto a su propio cuerpo. Muchas personas en la población parecen haber experimentado al menos una EFC: algunas encuestas tienen índices de respuesta positiva del 15\% (por ejemplo, Blackmore, 1984, Gómez Montanelli \& Parra 2008), y en estudiantes es del 25\% (Parra, 2013). Algunos estudios han mostrado una fuerte relación entre las experiencias fuera del cuerpo y variables psicológicas, especialmente la esquizotipia (McCreery y Claridge, 1995), las dimensiones del modelo factorial (como el NEO-PI-R) (Alvarado, Zingrone y Dalton, 1996), la absorción y experiencias disociativas (Parra \& Argibay, 2012). Estudios más recientes han examinado las bases neurofisiológicas subyacentes de la experiencia, lo cual ha revelado el origen neurofuncional de la experiencia de autoscopia o "verse a sí mismo" (Blanke, Landis, Spinelli, \& Seeck, 2004), el procesamiento mental durante la experiencias fuera del cuerpo (Blanke et al., 2005), y su relación con otras experiencias "disociativas", tales como las experiencias cercanas a la muerte (Blanke \& Dieguez, 2009) que ocurren en contexto médico y como reacción frente a la proximidad a la muerte.

Otras personas experimentan en forma definida un "yo exteriorizado", un cuerpo parasomático, o un "cuerpo astral" de acuerdo a la literatura de esoterismo o new age 
(para una revisión ver Alvarado \& Zingrone, 1999). La incidencia del cuerpo parasomático varía ampliamente, del 15 al 84\%, más del 90\% de las EFC son visuales a veces sólo visuales, y otras personas pueden controlar sus EFC (Irwin, 2004), es decir, manipulado hacia un determinado resultado, o simplemente ser una experiencia producto de la imaginación (Marsh, 2010). Irwin (2004) también examinó la experiencia en relación al concepto de absorción de Auke Tellegen, que se describe como la capacidad de estar "absorbido" o atento a representaciones imaginarias (Tellegen y Atkinson, 1974). Durante estos episodios, los individuos se vuelven totalmente concentrados en su experiencia, con "un compromiso total de sus recursos perceptuales, motrices, imaginativos e ideacionales a una representación única del objeto atencional" (Tellegen y Atkinson, 1974, p. 269). Irwin (2004) sostiene que las personas que han tenido experiencias extra corporales puntúan alto en absorción, es decir, son más susceptibles a las técnicas experimentales de inducción que aquellos que puntúan bajo. La relación positiva entre la EFC y la absorción es el primer vínculo que debe establecerse entre las EFC y disociación (Alvarado y Zingrone, 1997), de hecho la absorción es una de las experiencias disociativas más comunes.

Una evidencia adicional sugiere que las personas que han tenido experiencias fuera del cuerpo tienden a tener mayor nivel de actividad imaginativa/fantasía, lo cual es consistente con una personalidad propensa a la fantasía (Blackmore, 1978). La EFC podría ser una fantasía alucinatoria en individuos susceptibles a alucinar. También es coherente con la idea de que las personas que están más atentas a sus procesos mentales pueden ser más abiertos a experimentar EFC (Irwin, 2004) o recuerdan fantasías infantiles. La capacidad de absorción también es compatible con las prácticas de meditación y los sueños lúcidos, y personas que son propensas a fantasear, muestran mayor absorción, personalidad curiosa e intelectual y son emocionalmente más estables (Irwin, 2004).

Otro inconveniente en la evaluación de ciertas anomalías perceptuales que se 
extrapola del contexto de la psiquiatría clínica es su excesiva dependencia a ciertos fenómenos alucinatorios que se producen bajo modalidades visuales y auditivas. Asimismo, las alteraciones de la intensidad sensorial, más que la experiencia de fenómenos perceptuales discretos, por lo general no están presentes en las escalas de uso clínico. Otro legado de la psiquiatría clínica es la falta de investigación de anomalías perceptuales asociadas a alteraciones del lóbulo temporal, creencias y experiencias paranormales, así como fenómenos perceptuales anómalos en individuos no clínicos (Persinger y Makarec, 1987). Por lo tanto, sería necesario el empleo de una escala más eficaz y completa capaz de medir un rango de experiencias sensoriales que abarque tanto a muestras clínicas como no clínicas.

Por ejemplo, Bell, Halligan y Ellis (2006) diseñaron la Escala Cardiff de Percepciones Anómalas (CAPS) para medir anomalías perceptuales. Esta escala es independiente del contexto psiquiátrico convencional e incluye a determinadas experiencias subjetivas dentro del espectro de diferentes formas de percepción subjetiva (por ejemplo, saber que un percepto no está "realmente alli", el percepto parece extraño o inusual, o el percepto no es compartido como experiencia sensorial). Por otra parte, la CAPS incluye temas relacionados a distorsiones en la intensidad de percepción, a experiencias en todas las modalidades sensoriales normales, y experiencias sensoriales típicamente relacionadas con las alteraciones del lóbulo temporal.

Finalmente, el concepto de "límite fino" se refiere a la conexión de procesos psicológicos que refleja un estilo de pensamiento en escala de grises. La transliminalidad es "la tendencia del material psicológico a cruzar los umbrales desde dentro o hacia afuera de la conciencia" (Thalbourne y Houran, 2000, p. 861). El constructo de transliminalidad está compuesto por el concepto de absorción, propensión a la fantasía, ideación mágica, creencias paranormales, experiencias místicas, e hiperestesia, (la "hipersensibilidad a estímulos del medio ambiente", Thalbourne, 1999, p. 403). La transliminalidad sugiere que la fuente inmediata de 
nuestras percepciones no son nuestros ojos y oídos, sino más bien la conciencia subliminal: las percepciones que se procesan primero en un nivel inconsciente (y a veces externamente) luego se presentan, por lo general rápidamente, a lo largo de un espectro o "umbral" hacia la conciencia. Puntuaciones elevadas transliminalidad se presentan en aquellos que se consideran psíquicos o quienes desempeñan roles como chamanes o curanderos (Krippner, Wickramasekera y Tartz, 2001). Sherwood y Milner (2005) también concluyen que "la tendencia a tener experiencias psíquicas [...] podría ser un componente clave del constructo de límite" (p.376). El concepto de límite es valioso en cuanto a la comprensión de ciertos factores que subyacen a una variedad de experiencias psíquicas, como ver el aura. Respecto a experiencias anómalas, Thalbourne (1999) señala que "la esquizotipia representa probablemente el concepto empíricamente más cercano a la transliminalidad" (p. 20).

Este estudio tiene como objetivo comparar a un grupo de individuos que experimentaron EFC y un grupo control (sin EFC) usando tres cuestionarios psicológicos. Es importante reconocer que el estudio de las diferencias individuales de estas personas es importante no por otra razón que la de examinar su funcionamiento psicológico a un fenómeno tradicionalmente asociado a ciertas tradiciones esotéricas. En consecuencia, aquí se plantean tres hipótesis específicas: Las personas que tienen experiencias fuera del cuerpo tienen mayor frecuencia de (1) experiencias cognitivas anómalas (medidas por la CAPS de Bell), (2) mayor transliminalidad, (3) y límites más finos (puntuaciones bajas) en comparación con un grupo control (sin EFC).

\section{Método}

\section{Participantes}

La muestra estuvo compuesta por 211 participantes (159 mujeres 52 hombres), rango etario entre 18 a 83 años de edad $(M=44,92 ; D T=13,29)$, con buen nivel cultural y creyentes en lo paranormal, reclutados a través de medios de comunicación y una lista 
de correo electrónico de estudiantes interesados en lo paranormal y temas New Age. El anuncio también se colocó en una página web (www.alipsi.com.ar). La participación fue voluntaria y nadie recibió ninguna paga.

\section{Experiencias fuera del cuerpo}

La pregunta que releva la experiencia fuera del cuerpo era:

¿Alguna vez has tenido una experiencia en la que ha sentido que usted se localizaba fuera o lejos de su cuerpo físico, es decir, la sensación de que tu conciencia, mente o conciencia se encontraba en un lugar diferente de tu cuerpo físico? (En caso de duda, por favor, responda no). El rango de respuesta debe incluir los últimos seis meses.

La pregunta proviene de la versión en inglés Anomalous/Paranormal Experiences Inventory (Pekala, Kumar, \& Cummings, 1992). La pregunta examina dos dimensiones de la experiencia: frecuencia e impacto (emocional) positivo o negativo (1-7, siendo 7 el más alto). Dos datos adicionales fueron sexo, edad y grado de espiritualidad $(0=$ no soy espiritual a 5 = estoy muy espiritual).

Los participantes que respondieron "si" (Una vez, A veces o Múltiples veces) se agruparon como "EFC" ( $n=100,47 \%)$ y participantes que respondieron "no" se agruparon como "no EFC" ( $\mathrm{n}=111,53 \%$ ) (ver Tabla 1). La mayoría indicó haber tenido experiencias personales, tales como presentimientos $(58 \%)$, recuerdo de sueños $(50,8 \%)$, ver el aura $(34,7 \%)$ y otras experiencias paranormales $(38,3 \%)$.

\section{Diseño y Materiales}

La Escala Cardiff de Percepciones Anómalas (Bell, Halligan \& Ellis, 2006) es una escala autoadministrable que consta de 32 ítems diseñada para evaluar anomalías perceptuales tales como cambios en los niveles de intensidad sensorial, distorsión perceptual, desbordamiento sensorial, y alucinaciones. Se pidió a los participantes que calificaran cada ítem indicando n su respuesta No (0) o Si (1). Una puntuación alta indica mayor número de anomalías perceptuales en un rango de de 0 (nada) a 32 (lo 
más alto). La confiabilidad interna del CAPS es buena, con un coeficiente alfa de Cronbach de 0,87. La fiabilidad test-retest también se ha encontrado aceptable (Bell, Halligan \& Ellis, 2006).

El Escala de Transliminalidad-Revisada es una escala autoadministrable que presenta 29 ítems en formato dicotómico verdadero/falso (Thalbourne, 1999). Puntuación alta de transliminalidad involucra ciertas experiencias paranormales, experiencias místicas, creatividad, ideación mágica, absorción, propensión a la fantasía, hipersensibilidad a estímulos sensoriales, y una actitud positiva hacia la interpretación de los sueños. La escala de transliminalidad se ha administrado a grandes muestras en una variedad de contextos y culturas, de modo que hay correlaciones débiles, moderadas, y fuertes. Según Hartmann, Harrison y Zborowski (2001) altos niveles de transliminalidad están fuertemente correlacionados con límites "finos".

El Cuestionario de Límites (BQ) es una escala autoadministrable compuesta por 138 ítems que incluye diferentes dimensiones de límite (Barbuto y Plummer, 2000), dividida en 14 categorías: Tipo de límite, Dormir/vigilia/sueño, Experiencias inusuales, Pensamientos/sentimientos/estados de ánimo, Niñez/Adolescencia/Adultez, Relaciones interpersonales, Sensibilidad, Exactitud/Precisión/Límite, Opiniones acerca de organizaciones, Ropa, Opiniones acerca de niños y otras personas, Opiniones sobre personas, naciones y grupos, y Opiniones acerca de la belleza y la verdad, y Experiencias Paranormales. El formato de respuesta para cada pregunta va desde 0 (nada) a 4 (mucho). Aproximadamente dos tercios de los ítems están redactados de manera que "Totalmente de acuerdo" representa un límite "fino" y el resto de los ítems están redactados de manera que Totalmente en desacuerdo indica un límite "grueso". El BQ tiene buena fiabilidad test-retest (más de seis meses, $r=.77$ en dos muestras).

\section{Procedimiento}

Los tres cuestionarios fueron entregados bajo el pseudo-título de Cuestionario de Experiencias Psicológicas, en orden contrabalanceado, para evitar sesgos en sus 
respuestas. Se dió en un solo sobre a cada participante, quienes recibieron información sobre el objetivo del estudio (pero no las hipótesis) y fueron invitados a completar las tres escalas de forma voluntaria y anónima, en una sola sesión.

\section{Análisis}

En primer lugar, se llevó a cabo un contraste de hipótesis sobre la normalidad de las variables mediante un análisis de Kolmogorov-Sminoff $(p<0,001)$. A partir de los valores obtenidos, se asumió una distribución asimétrica de las puntuaciones de los instrumentos, en consecuencia, se utilizó estadística no paramétrica (para correlacionar Rho de Spearman y para comparar U de Mann-Whittney). Para contrarrestar el problema de correlaciones múltiples, se aplicó también el método Bonferroni de corrección, donde se estableció un nivel de significación de $<.004$, a una cola.

\section{Resultados}

Tabla 1

Frecuencia e impacto emocional de individuos con EFC por género

\begin{tabular}{lllll}
\hline & & $\begin{array}{l}\text { Varones } \\
(\boldsymbol{n}=52)\end{array}$ & $\begin{array}{l}\text { Mujeres } \\
(\boldsymbol{n}=\mathbf{1 5 9})\end{array}$ & Total \\
\hline \multirow{3}{*}{ Frequencia } & Nunca & $25(48,1 \%)$ & $86(54,1 \%)$ & $111(52,6 \%)$ \\
& Una vez & $5(9,6 \%)$ & $29(18,2 \%)$ & $34(16,1 \%)$ \\
& A veces & $20(38,5 \%)$ & $42(26,4 \%)$ & $62(29,4 \%)$ \\
\hline Impacto & Múltiples veces & $2(3,8 \%)$ & $2(1,3 \%)$ & $4(1,9 \%)$ \\
Emotional & Media (DT) ${ }^{(1)}$ & $1,77(2,48)$ & $1,47(2,11)$ & $1,54(2,23)$ \\
& & & &
\end{tabular}

(1) 0 = Negativo o displacentera a 7 = Positiva o placentera. 
Tabla 2

Comparación entre Individuos Con y Sin EFC en Transliminalidad, Límite Fino y Experiencias Anómalas

\begin{tabular}{|c|c|c|c|c|c|c|}
\hline \multirow[b]{3}{*}{ Variables } & \multicolumn{4}{|c|}{ Grupos } & \multirow[b]{3}{*}{$\mathrm{z}^{*}$} & \multirow[b]{3}{*}{$e_{s}^{* *}$} \\
\hline & \multicolumn{2}{|c|}{$\operatorname{Sin} E F C^{(1)}$} & \multicolumn{2}{|c|}{ Con EFC ${ }^{(2)}$} & & \\
\hline & Media & DT & Media & DT & & \\
\hline Intensidad Sensorial & 1,68 & 1,53 & 2,18 & 1,42 & 2,53 & 0,16 \\
\hline Experiencia Sensorial no Compartida & 1,50 & 1,32 & 2,27 & 1,29 & 4,12 & 0,28 \\
\hline Experiencia & 0,92 & 1,11 & 1,45 & 1,26 & 3,33 & 0,21 \\
\hline Compartida & & & & & & \\
\hline Experiencia Sensorial de Origen Inexplicable & 2,21 & 1,52 & 3,17 & 1,55 & 4,50 & 0,29 \\
\hline Distorsión corporal & 0,44 & 0,74 & 0,94 & 1,05 & 3,83 & 0,26 \\
\hline Alucinaciones Verbales & 0,58 & 0,81 & 0,97 & 0,07 & 3,12 & 0,32 \\
\hline Desbordamiento Sensorial & 0,71 & 0,71 & 0,92 & 0,72 & 2,12 & 0,14 \\
\hline Escuchar Pensamientos Exteriorizados & 0,38 & 0,59 & 0,36 & 0,50 & 0,10 & 0,01 \\
\hline Lóbulo Temporal & 1,50 & 1,04 & 2,07 & 1,16 & 3,40 & 0,25 \\
\hline Escala Cardiff & 9,92 & 6,79 & 14,33 & 7,16 & 4,41 & 0,30 \\
\hline Dormir / vigilia / sueño & 13,10 & 7,33 & 14,88 & 8,15 & 1,38 & 0,11 \\
\hline Experiencias inusuales & 16,60 & 9,00 & 23,00 & 7,59 & 5,50 & 0,35 \\
\hline $\begin{array}{l}\text { Pensamientos / sentimientos / estados de } \\
\text { ánimo }\end{array}$ & 18,42 & 9,79 & 25,08 & 8,62 & 5,11 & 0,33 \\
\hline Niñez / Adolescencia / Adultez & 9,71 & 3,90 & 11,00 & 3,79 & 2,16 & 0,16 \\
\hline Relaciones interpersonales & 21,87 & 5,02 & 22,17 & 4,34 & 1,42 & 0,03 \\
\hline Sensibilidad & 13,26 & 3,26 & 13,72 & 3,10 & 0,96 & 0,07 \\
\hline Exactitud / Precisión / Límite & 17,24 & 4,92 & 17,82 & 5,23 & 0,62 & 0,05 \\
\hline Ropa & 31,40 & 6,34 & 32,83 & 6,83 & 1,43 & 0,10 \\
\hline
\end{tabular}


Opiniones acerca de niños y otras personas $\quad \begin{array}{llllll}21,98 & 4,59 & 23,13 & 4,75 & 1,90 & 0,12\end{array}$

Opiniones acerca de organizaciones

$21,54 \quad 3,87 \quad 20,93 \quad 5,08 \quad 0,43 \quad 0,06$

Opiniones sobre personas, naciones y grupos

$27,90 \quad 6,14 \quad 28,17 \quad 6,09 \quad 0,12 \quad 0,02$

Opiniones acerca de la belleza y la verdad

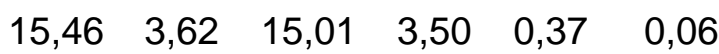

Experiencias Paranormales

$8,67 \quad 5,33 \quad 13,45 \quad 5,46 \quad 5,76 \quad 0,40$

Límite fino (Total)

$\begin{array}{lllll}237,15 & 40,06 & 260,91 & 40,45 & 4,33 \quad 0,28\end{array}$

Transliminalidad

$\begin{array}{llllll}13,22 & 4,85 & 9,83 & 4,97 & 4,49 & 0,32\end{array}$

Notas. EFC = Experiencia Fuera del Cuerpo; DT = Desvío Típico.

(1) Experiencias Fuera del Cuerpo $n=111$; (2) Sin Experiencias Fuera del Cuerpo $n=$ 100

${ }^{*} p<.05 ;{ }^{* *} p<.01 ;{ }^{* * *} p<.001$ ( $p$ ajustada). Estadística no paramétrica $U$ de Mann-

Whittney.

** Efecto de Magnitud.

Tabla 3

Correlaciones entre Espiritualidad, Impacto Emocional, Transliminalidad, Experiencias Anómalas, y Límite Fino

$\begin{array}{lllll}1 & 2 & 3 & 4 & 5\end{array}$

1. Espiritualidad

2. Impacto Emocional , 11

3. Transliminalidad $-, 19^{*}-, 29^{* * *} \quad-$

4. Experiencias Anómalas , 06

, $27^{* * *}$

$-, 59^{* * *}$

5. Límite Fino

, $22^{* *}$

, $31^{\star \star *}$

$-, 67^{* \star *}$

, $56^{* *}-$

${ }^{*} p<.05 ;{ }^{* \star} p<.01 ;{ }^{* \star *} p<.001$.

La hipótesis 1 era que individuos con EFC puntuarían más alto en experiencias anómalas (medido por el CAPS de Bell), la cual se confirmó: la media de EFC fue significativamente mayor que para los no EFC (ver Tabla 2). También puntuaron alto 
en las subescalas Intensidad sensorial, Experiencia sensorial no compartida, Experiencia sensorial distorsionada, Experiencia sensorial de una fuente inexplicable, Distorsión de la forma de propio cuerpo y del mundo externo, Alucinaciones verbales, Inundación sensorial, y Lóbulo temporal.

La hipótesis 2 era que individuos con EFC tenderían a mostrar límite fino, lo cual se confirmó: la media de los EFC fue significativamente más baja (hacia el "más fino") que los no EFC (ver Tabla 2). EFC también puntuaron bajo en las subescalas Experiencias inusuales, Pensamientos-Sentimientos-Estados de ánimo, NiñezAdolescencia-Edad adulta, y Experiencias paranormales.

La hipótesis 3 era que individuos con EFC puntuarían alto en Transliminalidad, lo cual se confirmó: la media de los EFC fue significativamente mayor que los no EFC (ver Tabla 2).

Una serie de correlaciones exploró la relación entre Transliminalidad, Experiencias anómalas, Límite fino, y también Espiritualidad e Impacto emocional de las EFC. Se encontraron ocho $(80 \%)$ de diez correlaciones: Personas que tenían límites más finos tienden a puntuar alto en Espiritualidad $(R h o=.22)$, Impacto emocional $(R h o=.31)$, Transliminalidad $(R h o=.67)$, y Experiencias anómalas $($ Rho $=.56)$. Personas que puntuaron alto en Experiencias anómalas tienden a puntuar más alto en Transliminalidad (Rho $=.27)$. Personas que obtuvieron puntuación más alta en Transliminalidad tienden a puntuar ligeramente más bajo en Espiritualidad ( $R h o=.19$ ), e Impacto emocional $(R h o=.25)($ Tabla 3$)$.

Se empleó una regresión logística binaria para evaluar cuál es el mejor predictor de la experiencia fuera del cuerpo mediante el método "Enter". Los resultados del mejor modelo encontró que las experiencias anómalas era el mejor predictor entre individuos con $E F C$ y $\sin E F C\left[\beta=.056, d f=1, p=0,05 ; R^{2}=.13\right]$, pero sólo en un grado débil. El resto de las variables no aportó nada a la predicción. Los análisis de las medida de impacto emocional $(M=2,44)$ no resultó significativo. Sólo si Experiencias anómalas 
(CAPS) se excluye de la regresión, Transliminalidad resulta el mejor predictor $[\beta=$ $0,11$, Wald $=6,83, \mathrm{df}=1, p=.009]$ con una $\beta$ más alta. Esto sugiere que la variable Transliminalidad puede estar afectando la diferencia entre ambos grupos.

\section{Discusión}

El presente estudio examinó las diferencias entre personas que tienen experiencias fuera del cuerpo y quienes no en las medidas de experiencias anómalas, transliminalidad y límite fino. El análisis de los datos confirmó las tres hipótesis. Personas que han tenido tales experiencias tienden a tener mayor frecuencia de experiencias sensoriales de fuente inexplicable (por ejemplo, sensaciones extrañas en el cuerpo, sonidos o formas inusuales), experiencias sensoriales no compartidas (por ejemplo, ver cosas que otros no pueden), distorsión del propio cuerpo y del mundo exterior (por ejemplo, la sensación de que sus miembros podrían no ser propios o no estar conectados al cuerpo), experiencia sensorial distorsionada (por ejemplo, sensaciones inusuales o extrañas en el cuerpo), experiencias relacionadas con el lóbulo temporal (por ejemplo, distorsión temporal, sensación de estar siendo elevado), alucinaciones verbales (por ejemplo, voces diciendo palabras o frases), y desbordamiento sensorial (por ejemplo, dificultad para distinguir sensaciones).

La variable transliminalidad, que se compone de absorción, propensión a la fantasía, ideación mágica, creencias paranormales, experiencia mística, e hiperestesia (esto es hipersensibilidad a estímulos del entorno, ver Thalbourne, 1999) resultó la más predictora de la EFC, de modo que aquellos cuya conciencia está "en ebullición" son propensos a experimentar imaginería sensorial más rápida e intensa. Además, personas que tuvieron experiencias fuera del cuerpo también experimentaron mayor frecuencia de experiencias inusuales (por ejemplo, experiencias de dejá vu), pensamientos, sentimientos, y estados de ánimo (por ejemplo, "no sé si estoy pensando o sintiendo"), sentimientos relacionados con su infancia y otras experiencias 
paranormales. Transliminalidad, experiencias anómalas, límite fino, espiritualidad e impacto emocional también están altamente interrelacionadas, por ejemplo, personas que tienen límites más finos tienden a ser más espirituales. La transliminalidad se correlaciona positivamente con el límite fino (Sherwood y Milner, 2005), y la esquizotipia (Thalbourne, 1999).

En general los "sistemas finos" son más propensos a experimentar fenómenos inusuales, y algunas de sus formas están más asociadas a las de ciertas experiencias anómalas. Braithwaite, Samson, Apperly, Broglia, and Hulleman (2011) llevaron a cabo dos estudios sobre experiencias fuera del cuerpo en población normal empleando la escala Cardiff (Bell, Halligan, \& Ellis, 2006) y encontraron que estas personas habían experimentado significativamente más anomalías cognitivas principalmente asociadas a la inestabilidad del lóbulo temporal y al procesamiento de la distorsión corporal en comparación con un grupo control.

Los datos que confirman que las personas con experiencias fuera del cuerpo tienen más experiencias anómalas, transliminalidad y límite "fino" también guardan coherencia conceptual con aquellos estudios que han encontrado que la propensión a la fantasía parece ser predictora de ciertas experiencias paranormales (para una revisión, ver Parra, 2010). Otros estudios también sugieren que la EFC se relaciona con procesos cognitivos relacionados con alucinaciones visuales y táctiles, y propensión a la fantasía (Alvarado y Zingrone, 1997). Por tales razones, la experiencia fuera del cuerpo, como una experiencia humana, debe ser mejor estudiada. Siguiendo a Irwin (2004, p. 10) "estas experiencias incluyen una amplia gama de diferentes dimensiones y hay muchos aspectos de éstas que deben estudiarse" además de determinar si hay algún presunto proceso paranormal subyacente. 


\section{Referencias}

Alvarado, C. S., \& Zingrone, N. (1997). Out-of-body experiences and dissociation. En R. Wiseman (Ed.), Parapsychological Association 40 ${ }^{\text {th }}$ Annual Convention: Proceedings of presented papers, (pp. 11-25). Brighton, UK.

Alvarado, C. S., \& Zingrone, N. L. (1999). Out-of-body experiences among readers of a Spanish New Age magazine. Journal of the Society for Psychical Research, 63, $65-85$.

Alvarado, C. S., Zingrone, N. L., \& Dalton, K. (1996). Out-of-body experiences, psi experiences, and the big five: Relating the NEO-PI-R to the experience claims of experimental participants. Parapsychological Association $39^{\text {th }}$ Annual Convention: Proceedings of presented papers.

Barbuto, J. \& Plummer, B. (2000). Mental boundaries and Jung's psychological types: A profile analysis. Journal of Psychological Type, 54, 17-21.

Bell, V, Halligan, P.W., \& Ellis, H.D. (2006). The Cardiff Anomalous Perceptions Scale (CAPS): A New Validated Measure of Anomalous Perceptual Experience. Schizophrenia Bulletin, 32, 366-377.

Blackmore, S. (1978). Parapsychology and out-of-the-body experiences. London: Transpersonal Books / Society for Psychical Research.

Blackmore, S. J. (1984). A postal survey of OBEs and other experiences. Journal of the Society for Psychical Research, 52, 225-244.

Blanke, O. \& Dieguez, S. (2009). Leaving Body and Life Behind: Out-of-Body and Near-Death Experience". En S. Laureys, S.; G. Tononi (Ed.) The Neurology of Consciousness: Cognitive Neuroscience and Neuropathology. (pp. 132-234) San Diego, CA: Elsevier.

Blanke, O., Landis, T., Spinelli, L., \& Seeck, M. (2004). Out-of-body experience and autoscopy of neurological origin. Brain, 127(2), 243-258

Blanke, O., Mohr, C., Michel, C. M., Pascual-Leone, A., Brugger, P., Seeck, M., ...Thut, 
G. (2005). Linking out-of-body experience and self-processing to mental ownbody imagery at the temporoparietal junction. Journal of Neuroscience: The Official Journal of the Society for Neuroscience, 25(3), 550-557.

Braithwaite, J.J., Samson, D., Apperly, I.; Broglia, E., \& Hulleman, J. (2011). Cognitive correlates of the spontaneous out-of-body experience (OBE) in the psychologically normal population: evidence for an increased role of temporallobe instability, body-distortion processing, and impairments in own-body transformations. Cortex, 47(7), 839-853.

Gómez Montanelli, D.E. \& Parra, A (2008). Are spontaneous anomalous/paranormal experiences disturbing?: A survey among under-graduate stundents. International Journal of Parapsychology, 13, 1-14.

Hartmann, E., Harrison, R., \& Zborowski, M. (2001).Boundaries in the Mind: Past Research and Future Directions. North American Journal of Psychology, 3, 347368.

Irwin, H.J. (2004). An introduction to Parapsychology (4⿳亠丷⿵冂丶 McFarland.

Krippner, S., Wickramasekera, I., \& Tartz, R. (2001). Scoring thick and scoring thin: The boundaries of psychic claimants. Journal of Subtle Energy, 11(1), 43-61.

Marsh, M. (2010). Out-of-body and near-death experiences: Brain-state phenomena or glimpses of immortality? Oxford \& New York: Oxford University Press.

McCreery, C., \& Claridge, G. (1995). Out-of-the body experiences and personality. Journal of the Society for Psychical Research, 60, 129-148.

Parra, A. (2010) Out-of-body experiences and hallucinatory experiences: A psychological approach. Imagination, Cognition and Personality, 29(3), 211224.

Parra, A. (2013). Cognitive and emotional empathy in relation to five paranormal/anomalous experiences. North American Journal of Psychology, 
15(3), 405-412.

Parra, A. \& Argibay, J.C (2012). Dissociation, absorption, fantasy proneness and sensation-seeking in psychic claimants. Journal of the Society for Psychical Research, 76, 193-203.

Pekala, R., Kumar, V.K. \& Cummings, J. (1992). Types of high hypnotically susceptible individuals and reported attitudes and experiences of the paranormal and the anomalous. Journal of the American Society for Psychical Research, 86, 135150.

Persinger, M.A. \& Makarec, K. (1987). Temporal lobe epileptic signs and correlative behaviors displayed by normal populations. Journal of Genetic Psychology, $114,179-195$.

Sherwood, S.J. \& Milner, M. (2005). The relationship between transliminality and boundary structure subscales. Imagination, Cognition and Personality, 24(4), 369-378.

Tellegen, A., \& Atkinson, G. (1974). Openness to absorbing and self altering experiences ("absorption"), a trait related to hypnotic susceptibility. Journal of Abnormal Psychology, 83, 268-277.

Thalbourne, M.A. (1999). Transliminality: A review. International Journal of Parapsychology, 11(2), 1-34.

Thalbourne, M.A., \& Houran, J. (2000). Transliminality, the mental experience inventory and tolerance of ambiguity. Personality and Individual Differences, 28, 853-863.

\section{Formato de citación}

Parra, A. (2018). Experiencias fuera del cuerpo: una evaluación del constructo de transliminalidad y límite "fino" como anomalía cognitivo-perceptual. Psicología, Conocimiento y Sociedad, 8(1), 100-116. doi: http://dx.doi.org/10.26864/PCS.v8.n1.5 